ANNALES

POLONICI MATHEMATICI

LVIII.1 (1993)

\title{
A note on solutions of semilinear equations at resonance in a cone
}

\author{
by BOGdAN PRZERADZKI (Łódź)
}

\begin{abstract}
A connection between the Landesman-Lazer condition and the solvability of the equation $L x=N(x)$ in a cone with a noninvertible linear operator $L$ is studied. The result is based on the abstract framework from [5], applied to the existence of periodic solutions of ordinary differential equations, and compared with theorems by Santanilla (see [7]).
\end{abstract}

1. Introduction. The question of the solvability of the equation $L x=$ $N(x)$, where $L$ is a linear noninvertible operator and $N$ a nonlinear and completely continuous operator, has a twenty years' history. $L$ is usually assumed to be Fredholm of index zero, and the so-called Landesman-Lazer conditions [2], [9], i.e. some integral relations between functions spanning ker $L$ and certain asymptotic characteristics of $N$, guarantee the existence of solutions. The problem can be studied from several abstract points of view. The most general one seems to be the approach due to J. Mawhin [3] but we shall consider another one developed in [4]-[6]. This needs the assumption that $L$ is a boundary point of the set of linear invertible operators. Our method lies in applying the continuation of $L$ instead of $N$. As a result, we obtain conditions of Landesman-Lazer type in an abstract form.

A next natural question is if there exists a solution of $L x=N(x)$ belonging to a fixed set $K$. This set is usually a cone for applicational reasons - as an example, we give the $n$-dimensional periodic problem

$$
x^{\prime}=f(t, x), \quad x(0)=x(1),
$$

where we seek for a nonnegative solution, i.e.

$$
K=\left\{x:[0,1] \rightarrow \mathbb{R}^{n} \mid x_{j}(t) \geq 0 \text { for } t \in[0,1], j=1, \ldots, n\right\} .
$$

This problem as well as similar ones were studied by Gaines and Santanilla [1] and then by Santanilla [7], [8].

1991 Mathematics Subject Classification: 34C25, 47H15.

Key words and phrases: nonnegative solutions, equations at resonance. 
Although they used abstract resonance theorems based on Mawhin's approach, their results can be obtained without these theorems, as Santanilla partially noticed in [7]. This is possible since the equation $L x=N(x)$ is equivalent to $(L-\alpha I) x=N(x)-\alpha x$ with $L-\alpha I$ invertible. This reduces the problem to the question of the existence of a fixed point for a completely continuous operator, which is solvable by means of the Leray-Schauder continuation method.

We shall say that a theorem giving the existence of a solution to $L x=$ $N(x)$ with $L$ noninvertible is a resonance theorem if its assertion cannot be obtained from the solvability of $L x-\alpha x=N(x)-\alpha x$ with a fixed $\alpha \neq 0$. The author knows that the question is subtle but he believes that the results given below are of resonance type. However, it should be stressed that our assumptions guaranteeing the nonnegative solvability of periodic problems are neither better nor worse than those of Santanilla.

2. General setting. Let $X, Y, Z$ be Banach spaces, $L:\left[\lambda_{0}, \lambda_{1}\right] \rightarrow$ $L(Y, Z)$ a continuous mapping of an interval into the space of all linear bouded operators from $Y$ into $Z$ such that $L(\lambda)$ is a linear homeomorphism for $\lambda \neq \lambda_{0}$ and $L\left(\lambda_{0}\right)$ is a Fredholm operator with a nontrivial kernel (of index zero). We shall assume that the inverse operators $G(\lambda)=L(\lambda)^{-1}$ have the form

$$
G(\lambda)=G_{0}(\lambda)+\sum_{j=1}^{n} c_{j}(\lambda)\left\langle u_{j}(\lambda), \cdot\right\rangle w_{j}(\lambda)
$$

where $G_{0}(\lambda) \in L(Z, Y), u_{j}(\lambda) \in Z^{*}, w_{j}(\lambda) \in Y$ are continuous functions of $\lambda$ having continuous extensions onto the whole interval $\left[\lambda_{0}, \lambda_{1}\right]$, and $c_{j}(\lambda)$ $\in \mathbb{R}$

$$
\lim _{\lambda \rightarrow \lambda_{0}^{+}}\left|c_{j}(\lambda)\right|=\infty .
$$

Suppose that $w_{j}\left(\lambda_{0}\right), j=1, \ldots, n$, are linearly independent and span ker $L\left(\lambda_{0}\right)$, and that

$$
\operatorname{Im} L\left(\lambda_{0}\right)=\bigcap_{j=1}^{n} \operatorname{ker} u_{j}\left(\lambda_{0}\right) .
$$

It follows that the systems $\left\{w_{j}(\lambda): j=1, \ldots, n\right\}$ and $\left\{u_{j}(\lambda): j=1, \ldots, n\right\}$ are linearly independent for $\lambda$ sufficiently close to $\lambda_{0}$. We can assume without loss of generality that this holds for $\lambda \in\left[\lambda_{0}, \lambda_{1}\right]$. Moreover, suppose that $G_{0}\left(\lambda_{0}\right)$ is a right inverse for $L\left(\lambda_{0}\right)$, i.e. $L\left(\lambda_{0}\right) G_{0}\left(\lambda_{0}\right) z=z$ for any $z \in$ $\operatorname{Im} L\left(\lambda_{0}\right)$.

Let $N: \quad X \rightarrow Z$ be a nonlinear continuous operator and $J: Y \rightarrow X$ a completely continuous linear injective operator (usually, it is an inclusion 
map). The resonance problem we shall examine is

$$
L\left(\lambda_{0}\right) y=N(J y) \text {. }
$$

It is equivalent to the system

$$
\begin{aligned}
& y=G_{0}\left(\lambda_{0}\right) N(J y)+\sum_{j=1}^{n} C_{j} w_{j}\left(\lambda_{0}\right), \\
& \left\langle u_{j}\left(\lambda_{0}\right), N(J y)\right\rangle=0 \quad \text { for } j=1, \ldots, n,
\end{aligned}
$$

where $C_{1}, \ldots, C_{n}$ are arbitrary real constants.

We restrict ourselves to the case of sublinear nonlinearity:

$$
\lim _{\|x\| \rightarrow \infty}\|N(x)\| /\|x\|=0 \text {. }
$$

From [5], we quote

THEOREM 1. If , for any $\left(x_{m}\right) \subset X$ such that $\left\|x_{m}\right\| \rightarrow \infty$ and $\left\|x_{m}\right\|^{-1} x_{m}$ $\rightarrow \sum_{j} d_{j} J w_{j}\left(\lambda_{0}\right)$, there exists $j_{0} \in\{1, \ldots, n\}$ such that

$$
\limsup _{m \rightarrow \infty} \operatorname{sgn} c_{j_{0}}(\lambda) d_{j_{0}}\left\langle u_{j_{0}}\left(\lambda_{0}\right), N\left(x_{m}\right)\right\rangle<0,
$$

then equation (2.3) has a solution.

Sketch of proof. The equations $x_{\lambda}=J G(\lambda) N\left(x_{\lambda}\right), \lambda \in\left[\lambda_{0}, \lambda_{1}\right]$, have solutions $x_{\lambda}$ by the Rothe fixed point theorem. Take $\lambda_{m} \rightarrow \lambda_{0}^{+}$. If $\left(x_{\lambda_{m}}\right)$ is bounded, then it contains a convergent subsequence by compactness arguments and its limit is the sought-for solution. If $\left\|x_{\lambda_{m}}\right\| \rightarrow \infty$, we can repeat the above arguments for $\left\|x_{\lambda_{m}}\right\|^{-1} x_{\lambda_{m}}$ to conclude that its subsequence tends to $\sum d_{i} J w_{i}\left(\lambda_{0}\right)$. It follows that

$$
c_{j}\left(\lambda_{m}\right)\left\langle u_{j}\left(\lambda_{m}\right), N\left(x_{\lambda_{m}}\right)\right\rangle
$$

has the same sign as $d_{j}$ for all $j=1, \ldots, n$, which contradicts (2.6).

Inequality (2.6) is called the Landesman-Lazer condition (L-L) and, in the case of the Dirichlet boundary value problem

$$
x^{\prime \prime}+m^{2} x=f(t, x), \quad x(0)=x(\pi)=0,
$$

it is equivalent to the classical Landesman and Lazer assumption (comp. $[5])$.

3. Why the L-L condition is inconsistent with cone preservation? We would like to find a solution of equation (2.3) such that $J y \in K$ where $K$ is a fixed cone in $X$, i.e.

$$
x_{1}, x_{2} \in K \Rightarrow x_{1}+x_{2} \in K, \quad x \in K, \lambda \geq 0 \Rightarrow \lambda x \in K,
$$

and $K$ is a closed subset of $X$. The method based on Section 2 needs the following additional assumptions: 
(i) $J w_{j}(\lambda) \in K$

(ii) $x \in K \Rightarrow J G_{0}(\lambda) N(\lambda) \in K, \operatorname{sgn} c_{j}(\lambda)\left\langle u_{j}(\lambda), N(x)\right\rangle \geq 0$ for $\lambda \in\left[\lambda_{0}, \lambda_{1}\right]$ and $j=1, \ldots, n$.

They are necessary for $J G(\lambda) N$ to be cone preserving mappings, so the solutions $x_{\lambda}=J G(\lambda) N\left(x_{\lambda}\right) \in K$, and thus, there exists a sequence $x_{\lambda_{m}} \rightarrow$ $x \in K$ with $x$ being a solution of our equation as in the proof of Theorem 1 . Since $\sum d_{i} J w_{i}\left(\lambda_{0}\right) \in K$ for $d_{1}, \ldots, d_{n} \geq 0$, we have

$$
\operatorname{sgn} c_{j}(\lambda)\left\langle u_{j}\left(\lambda_{0}\right), N\left(\sum d_{i} J w_{i}\left(\lambda_{0}\right)\right)\right\rangle \geq 0, \quad j=1, \ldots, n
$$

which contradicts the L-L condition.

We give a version of the resonance theorem for solutions in a fixed set which is a direct consequence of Theorem 1 .

THEOREM 2. If all assumptions of Theorem 1 are satisfied and $K$ is a subset of $X$ such that each solution of the system (2.4) belongs to $K$, then equation (2.3) has a solution $y$ with $J y \in K$.

We shall show how this theorem works in applications.

4. Periodic nonnegative solutions of first order differential equations. Let $f=\left(f_{1}, \ldots, f_{n}\right):[0,1] \times \mathbb{R}^{n} \rightarrow \mathbb{R}^{n}$ be a Carathéodory function, i.e. $f(t, \cdot): \mathbb{R}^{n} \rightarrow \mathbb{R}^{n}$ is continuous for a.e. $t \in[0,1]$ and $f(\cdot, x)$ is measurable for all $x$. Suppose that $f$ is sublinear:

$$
\|f(t, x)\| \leq a\|x\|^{\varrho}+b(t)
$$

where $a>0, \varrho \in[0,1)$ and $b \in L^{1}(0,1)$. It follows that the Nemytskiu operator $N(x)(t)=f(t, x(t))$ maps $L^{1}\left([0,1], \mathbb{R}^{n}\right)$ into itself and

$$
\lim _{\|x\|_{L^{1} \rightarrow \infty}}\|N(x)\|_{L^{1}} /\|x\|_{L^{1}}=0 .
$$

Let $K_{n}^{+}=\left\{x=\left(x_{1}, \ldots, x_{n}\right) \in \mathbb{R}^{n} \mid x_{i} \geq 0, i=1, \ldots, n\right\}$ and $K=\{\phi \in$ $L^{1}\left([0,1], \mathbb{R}^{n}\right) \mid \phi(t) \in K_{n}^{+}$for a.e. $\left.t \in[0,1]\right\}$. We look for a solution of the problem

$$
x^{\prime}=f(t, x), \quad x(0)=x(1), \quad x \in K .
$$

Theorem 3. Suppose that

$$
f_{j}\left(t, x_{1}, \ldots, x_{j-1}, 0, x_{j+1}, \ldots, x_{n}\right) \leq 0
$$

for $t \in[0,1], x_{1}, \ldots, x_{n} \geq 0, j=1, \ldots, n$, and, for all $j \in\{1, \ldots, n\}$,

$$
\inf _{x_{i} \geq 0, i \neq j} \int_{0}^{1} \liminf _{x_{j} \rightarrow \infty} f_{j}\left(t, x_{1}, \ldots, x_{n}\right) d t>0 .
$$

Then problem (4.3) has a solution. 
Proof. Define $g:[0,1] \times \mathbb{R}^{n} \rightarrow \mathbb{R}^{n}$ by

$$
g_{j}(t, x)=f_{j}\left(t,\left|x_{1}\right|, \ldots,\left|x_{j-1}\right|, x_{j},\left|x_{j+1}\right|, \ldots,\left|x_{n}\right|\right)
$$

for $t \in[0,1], x_{1}, \ldots, x_{n} \in \mathbb{R}, x_{j} \geq 0$, and

$$
g_{j}(t, x)=f_{j}\left(t,\left|x_{1}\right|, \ldots,\left|x_{j-1}\right|, 0,\left|x_{j+1}\right|, \ldots,\left|x_{n}\right|\right)-\sqrt{\left|x_{j}\right|}
$$

for $t \in[0,1], x_{j}<0$. It is obvious that $g$ is a Carathéodory function with property (4.1) and that the sets of solutions of (4.3) and of

$$
x^{\prime}=g(t, x), \quad x(0)=x(1), \quad x \in K,
$$

are the same. Hence we can study the second problem. The function $g$ has property (4.5) and, instead of (4.4),

$$
g_{j}(t, x)<0 \quad \text { for } x_{j}<0, \quad \lim _{x_{j} \rightarrow-\infty} g_{j}(t, x)=-\infty
$$

uniformly with respect to $t, x_{1}, \ldots, x_{j-1}, x_{j+1}, \ldots, x_{n}$.

Put $X=Z=L^{1}\left([0,1], \mathbb{R}^{n}\right), Y=\left\{\phi:[0,1] \rightarrow \mathbb{R}^{n} \mid \phi\right.$ is absolutely continuous $\left.\left(\phi^{\prime} \in L^{1}\right), \phi(0)=\phi(1)\right\}, J \phi=\phi, L(\lambda) \phi=\phi^{\prime}-\lambda \phi, \lambda_{0}=0$, $N(x)(t)=g(t, x(t))$. It is easy to see that all conditions of Section 2 are satisfied with

$$
\begin{aligned}
& G_{0}(\lambda) h(t)=e^{\lambda t} \int_{0}^{t} e^{-\lambda s} h(s) d s \\
& \quad-\left(e^{\lambda}-1\right)^{-1}\left(e^{\lambda}-e^{\lambda(t+1)}\right) \int_{0}^{1} e^{-\lambda s} h(s) d s, \\
& c_{j}(\lambda)=-\left(e^{\lambda}-1\right)^{-1} e^{\lambda}, \\
& \left\langle u_{j}(\lambda), h\right\rangle=\int_{0}^{1} e^{-\lambda s} h_{j}(s) d s, \\
& w_{j}(\lambda)(t)=(0, \ldots, 0,1,0, \ldots, 0) \quad(1 \text { in the } j \text { th place }),
\end{aligned}
$$

for $j=1, \ldots, n$. By (4.5), for $\left\|x_{m}\right\| \rightarrow \infty,\left\|x_{m}\right\|^{-1} x_{m} \rightarrow d \in \mathbb{R}^{n}$ in $L^{1}$ with $d_{j}>0$, and

$$
\left\langle u_{j}(0), N\left(x_{m}\right)\right\rangle>0 .
$$

On the other hand, by (4.6), this number is negative if $d_{j}<0$. It suffices to notice that $\operatorname{sgn} c_{j}(\lambda)=-1$ for any $j$ to get the L-L condition. 
System (2.4) has the following form:

$$
\begin{aligned}
& x(t)=\int_{0}^{t} g(s, x(s)) d s+\left(d_{1}, \ldots, d_{n}\right), \\
& \int_{0}^{1} g(s, x(s)) d s=0 .
\end{aligned}
$$

If, for a solution $x$ of this system, $x_{j}\left(t_{0}\right)<0$, then the function $x_{j}$ would have a negative minimum $x_{j}\left(t_{1}\right)<0$, while $0=x_{j}^{\prime}\left(t_{1}\right)=g_{j}\left(t_{1}, x\left(t_{1}\right)\right)<0$ from (4.6). Thus, all solutions of this system belong to $K$ and, by Theorem 2 , we obtain the assertion.

R e m a r k 1 . Taking an interval $\left[\lambda_{0}, 0\right]$ instead of $\left[0, \lambda_{1}\right]$, we have $\operatorname{sgn} c_{j}(\lambda)$ $=+1$, and inequalities (4.4), (4.5) can be reversed:

$$
\begin{gathered}
f_{j}\left(t, x_{1}, \ldots, x_{j-1}, 0, x_{j+1}, \ldots, x_{n}\right) \geq 0, \\
\sup _{x_{i} \geq 0, i \neq j} \int_{0}^{1} \limsup _{x_{j} \rightarrow \infty} f_{j}\left(t, x_{1}, \ldots, x_{n}\right) d t<0 .
\end{gathered}
$$

Remark 2. The assumptions of this theorem concerning $f$ are posed only on the boundary of the cone $K$ (and at infinity which can be treated as a boundary point of $K$ ), while in [7], $f$ should be controlled also in the interior of $K$. It is not clear if inequality (4.5) can be weakened to $\geq$.

Remark 3. In fact, $f$ can be defined only on $[0,1] \times K_{n}^{+}$and (4.1) is necessary only for $x \in K_{n}^{+}$. Moreover, one can consider the nonlinearity with linear growth after [5], [6], which means that $\varrho=1$ in (4.1). However, this restricts the problem to classical solutions with $f$ continuous, $X=Z=$ $C\left([0,1], \mathbb{R}^{n}\right), Y=\left\{\phi \in C^{1}\left([0,1], \mathbb{R}^{n}\right) \mid \phi(0)=\phi(1)\right\}$, since the sup-norms work better. If

$$
\gamma=\limsup _{\|x\| \rightarrow \infty} \sup _{t}\|f(t, x)\| /\|x\|<1,
$$

then assumptions (4.4) and (4.5) for all $j$ 's guarantee the solvability of (4.3).

5. Second order differential equations and their nonnegative solutions. We deal with the equation

$$
x^{\prime \prime}=f\left(t, x, x^{\prime}\right)
$$

where $f:[0,1] \times \mathbb{R}^{n} \times \mathbb{R}^{n} \rightarrow \mathbb{R}^{n}$ is a Carathéodory function such that

$$
\left\|f\left(t, x, x^{\prime}\right)\right\| \leq a_{1}\|x\|^{\varrho}+a_{2}\left\|x^{\prime}\right\|^{\varrho}+b(t)
$$

with $a_{1}, a_{2} \geq 0, \varrho<1$ and $b \in L^{1}(0,1)$. Now, the Nemytskiu operator

$$
N(x)(t)=f\left(t, x(t), x^{\prime}(t)\right)
$$


maps the Sobolev space $X=W^{1,1}\left([0,1], \mathbb{R}^{n}\right)$ of all functions $x:[0,1] \rightarrow \mathbb{R}^{n}$ integrable with the first derivative into $Z=L^{1}\left([0,1], \mathbb{R}^{n}\right)$, and $N: X \rightarrow Z$ is sublinear. We denote by $K_{n}^{+}$and $K$ the same cones as in Section 4 , but $K \subset W^{1,1}$ now. Notice that a solution in $K$ of equation (5.1) need not be a solution in $K$ of the corresponding first order system. We look for a 1-periodic solution of (5.1), i.e. the boundary conditions are

$$
x(1)-x(0)=0=x^{\prime}(1)-x^{\prime}(0),
$$

belonging to $K$.

THEOREM 4. If

$$
f_{j}\left(t, x_{1}, \ldots, x_{j-1}, 0, x_{j+1}, \ldots, x_{n}, y_{1}, \ldots, y_{n}\right) \leq 0
$$

for $t \in[0,1],\left(x_{1}, \ldots, x_{n}\right) \in K_{n}^{+},\left(y_{1}, \ldots, y_{n}\right) \in \mathbb{R}^{n}$ and $j=1, \ldots, n$, and, for each $j \in\{1, \ldots, n\}$,

$$
\inf _{y \in \mathbb{R}^{n}} \inf _{x_{i} \geq 0, i \neq j} \int_{0}^{1} \liminf _{x_{j} \rightarrow \infty} f_{j}(t, x, y) d t>0,
$$

then BVP (5.1)-(5.2) has a nonnegative solution.

Proof. Let $Y=\left\{\phi \in W^{2,1}\left([0,1], \mathbb{R}^{n}\right) \mid \phi(1)-\phi(0)=0=\phi^{\prime}(1)-\phi^{\prime}(0)\right\}$ where $W^{2,1}$ stands for the Sobolev space of functions integrable with the first and second derivatives. Let $L(\lambda) x=x^{\prime \prime}+\lambda x$ and let $g:[0,1] \times \mathbb{R}^{n} \times \mathbb{R}^{n} \rightarrow \mathbb{R}^{n}$ be a function replacing $f$, with the properties (5.4) and

$$
g_{j}(t, x, y)<0 \quad \text { for } x_{j}<0, \quad \lim _{x_{j} \rightarrow-\infty} g_{j}(t, x, y)=-\infty
$$

uniformly with respect to $t, x_{i} \geq 0$ for $i \neq j, y \in \mathbb{R}^{n}$. We do not find the Green operator explicitly as above since it is difficult to decompose it into regular $\left(G_{0}(\lambda)\right)$ and irregular parts. We can use the fact that the problem is self-adjoint and $G(\lambda)$ has the form given by the Hilbert-Schmidt theory:

$$
\begin{gathered}
G(\lambda)=\left(G^{1}(\lambda), \ldots, G^{n}(\lambda)\right), \\
G^{j}(\lambda) z=\sum_{m \in \mathbb{Z}}\left(\lambda-4 m^{2} \pi^{2}\right)^{-1}\left(z_{j}, e_{m}\right) e_{m}, \quad j=1, \ldots, n,
\end{gathered}
$$

where $(\cdot, \cdot)$ denotes the scalar product in $L^{2}(0,1), e_{m}(t)=\exp (2 i m \pi t)$ for $m \in \mathbb{Z}$. This is an integral operator with kernel

$$
\widetilde{G}^{j}(t, s ; \lambda)=\sum_{m \in \mathbb{Z}}\left(\lambda-4 m^{2} \pi^{2}\right)^{-1} e_{m}(t-s) .
$$

Obviously, it is a real continuous function if $\lambda \neq 4 m^{2} \pi^{2}$ and its $t$-derivative is also continuous. It follows that $G(\lambda)$ maps $Z$ into $Y$ and is the inverse of 
$L(\lambda)$. Put

$$
\begin{aligned}
G_{0}^{j}(\lambda) z & =\sum_{m \in \mathbb{Z} \backslash\{0\}}\left(\lambda-4 m^{2} \pi^{2}\right)^{-1}\left(z_{j}, e_{m}\right) e_{m}, \\
G_{0}(\lambda) & =\left(G_{0}^{1}(\lambda), \ldots, G_{0}^{n}(\lambda)\right), \\
c_{j}(\lambda) & =\lambda^{-1}, \quad\left\langle u_{j}(\lambda), z\right\rangle=\left(z_{j}, e_{0}\right), \quad w_{j}(\lambda)=e_{0},
\end{aligned}
$$

for $j=1, \ldots, n, J y=y, N(x)(t)=g\left(t, x(t), x^{\prime}(t)\right)$, and finally, $\lambda_{1}<0$.

If $\left\|x_{k}\right\| \rightarrow \infty,\left\|x_{k}\right\|^{-1} x_{k} \rightarrow d \in \mathbb{R}^{n},\left\|x_{k}\right\|^{-1} x_{k}^{\prime} \rightarrow 0$ in $L^{1}\left([0,1] ; \mathbb{R}^{n}\right)$ and $d_{j}<0$, we have

$$
\limsup _{k \rightarrow \infty} \operatorname{sgn} c_{j}(\lambda) d_{j}\left\langle u_{j}(0), N\left(x_{k}\right)\right\rangle=d_{j} \liminf _{k \rightarrow \infty} \int_{0}^{1} g_{j}\left(t, x_{k}(t), x_{k}^{\prime}(t)\right) d t<0
$$

by (5.5). If $d_{j} \geq 0$ for $j=1, \ldots, n$, then from (5.4) the lim sup is again negative for some $j$. The L-L condition holds.

On the other hand, equation (5.1) does not admit periodic solutions $x$ such that $x_{j}\left(t_{0}\right)<0$ for a certain $t_{0}$ and $j \in\{1, \ldots, n\}$, since such a function has a minimum $x_{j}\left(t_{1}\right)<0$ where $x_{j}^{\prime}\left(t_{1}\right)=0$ and $x_{j}^{\prime \prime}\left(t_{1}\right) \geq 0$, which contradicts (5.5)

All remarks to Theorem 3 except Remark 1 can be repeated here. The norm of the operator $J G_{0}(0)$ (necessary to find an estimate of $\gamma$, cf. [5]) cannot be easily calculated. One can consider a simpler case: $f$ independent of $x^{\prime}$, and

$$
\|f(t, x)\| \leq a\|x\|+b(t)
$$

where $b \in L^{2}(0,1)$. This enables one to work in $L^{2}\left([0,1], \mathbb{R}^{n}\right)$ where $\left\|J G_{0}(0)\right\|$ $=(2 \pi)^{-2}$.

Remark 4. All arguments can be repeated if the periodic boundary condition (5.2) is replaced by the Neumann condition:

$$
x^{\prime}(0)=0=x^{\prime}(1) .
$$

The corresponding Dirichlet problem $x(0)=0=x(1)$ is not of resonance type.

\section{References}

[1] R. E. Gaines and J. Santanilla, A coincidence theorem in convex sets with applications to periodic solutions of ordinary differential equations, Rocky Mountain J. Math. 12 (1982), 669-678.

[2] E. M. Landesman and A. C. Lazer, Nonlinear perturbations of linear elliptic boundary value problems at resonance, J. Math. Mech. 19 (1970), 609-623.

[3] J. L. Mawhin, Topological degree methods in nonlinear boundary value problems, CBMS Regional Conf. Ser. in Math. 40, Amer. Math. Soc., Providence, R.I., 1979. 
[4] B. Przeradzki, An abstract version of the resonance theorem, Ann. Polon. Math. 53 (1991), 35-43.

[5] -, Operator equations at resonance with unbounded nonlinearities, preprint.

[6] -, A new continuation method for the study of nonlinear equations at resonance, J. Math. Anal. Appl., to appear.

[7] J. Santanilla, Nonnegative solutions to boundary value problems for nonlinear first and second order ordinary differential equations, ibid. 126 (1987), 397-408.

[8] - Existence of nonnegative solutions of a semilinear equation at resonance with linear growth, Proc. Amer. Math. Soc. 105 (1989), 963-971.

[9] S. A. Williams, A sharp sufficient condition for solution of a nonlinear elliptic boundary value problem, J. Differential Equations 8 (1970), 580-586.

INSTITUTE OF MATHEMATICS

UNIVERSITY OF ŁÓDŹ

BANACHA 22

90-238 ŁÓDŹ, POLAND 